\title{
Super Heavy Elements - experimental developments
}

\author{
Gottfried Münzenberg ${ }^{1,2,3, *}$ \\ ${ }^{1}$ GSI Helmholtzzentrum für Schwerionenforschung mbH, Planckstr. 1, 64291 Darmstadt, Germany \\ ${ }^{2}$ Johannes Gutenberg Universität Mainz, 55122 Mainz, Germany \\ ${ }^{3}$ Manipal Centre for Natural Sciences, Manipal University, Manipal 576104, Karnataka, India
}

\begin{abstract}
With his theoretical work Walter Greiner, our mentor, pioneered super heavy element research and motivated us young scientists. He actively shaped the profile of GSI. We are happy that still during his lifetime we could prove some of his predictions: Fusion with magic nuclei paving the way to super heavy elements and the proof of the prediction of the nuclear species existing only by shell stabilization, super heavy elements. With the discovery of oganesson, $Z=118$, the heaviest element known today, we have come to the end of this era. New experimental developments will be discussed.
\end{abstract}

\section{Introduction}

Super-heavy element research was pioneered and strongly supported by Walter Greiner. In his early calculations he worked on the prediction of super heavy elements: atomic nuclei existing only by shell stabilisation in the sea of liquid-drop instability [1] and heavyelement production in reactions with the doubly magic nuclei, ${ }^{208} \mathrm{~Pb}$ and ${ }^{48} \mathrm{Ca}[2,3]$. At that time, when we started our experiments, it was not at all clear which reaction could be used to create heavy elements beyond element 106.

As member of the "Kernphysikalische Arbeitsgemeinschaft Hessen, KAH" Greiner shaped the research program of GSI, the Gesellschaft für Schwerionenforschung, $m b H$ in Darmstadt, at that time called "SILAB" (Fig. 1).

Main issues were the specifications for the accelerator to accelerate all ions of all elements of the periodic table and the production of new chemical elements and isotopes including Super Heavy Elements, SHE: The uranium beam as a rich source for new isotopes at the GSI fragment separator, and the new super heavy elements at SHIP. Both the basis for success of GSI.

*E-mail: G.Muenzenberg@gsi.de 


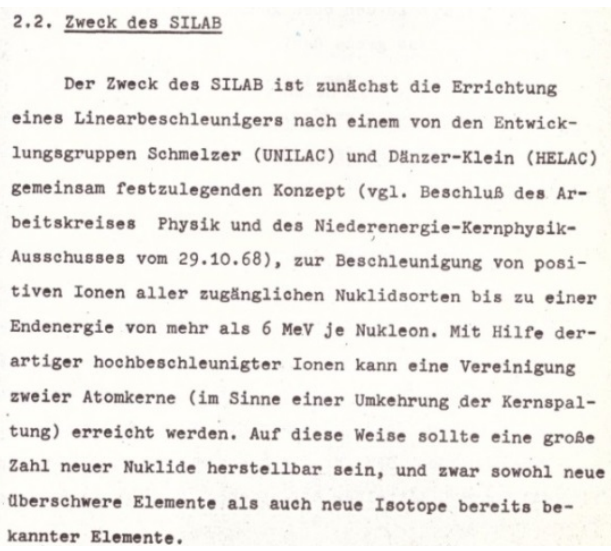

Fig. 1 Outline of the concept for the GSI accelerator and the research program taken from the SILAB proposal, Darmstadt 1969.

\section{Super heavy elements: the presence}

In March this year two milestones in SHE research were celebrated: The official announcement of the names of the heaviest known chemical elements by Natalya Tarasova, the president of, the "International Union of Pure and Applied Chemistry, IUPAC" [4]. Japan celebrated the new element 113, Nihonium in the Japanese Academy of science with a speech of Crown Prince Naruhito. Nihon is the original name of Japan. With nihonium the end of the cold fusion to create super heavy elements with lead or bismuth targets had been reached. Earlier, in March, an International Colloquium dedicated to the naming of elements 115 Moscovium, 117 Tennesine, and 118 Oganesson was held in the Russian Academy of Sciences, Moscow. The elements carry the names of Moscow region, where Dubna, the place of discovery, is located, the US state of Tennessee where Oak Ridge is located, the place of manufacturing the actinide targets, and Yuri Oganessian, "for his pioneering contributions to transactinoid elements research". With element 118 the synthesis of super heavy elements with actinide targets and ${ }^{48} \mathrm{Ca}$ beams, the era of synthesis of super heavy elements with doubly magic nuclei, as theoretically substantiated by Walter Greiner has come to its end. Now all chemical elements discovered so far are named officially. A complete overview on SHE research is given in a special issue of Nucl. Phys. A $[5]$.

\subsection{Discoveries}

At SHIP we experimentally proved the cold heavy ion fusion for SHE production and the idea of shell stabilized nuclei. First experiments to produce heavy elements in cold fusion reactions were performed by Oganessian. In irradiations of ${ }^{208} \mathrm{~Pb}$ and ${ }^{209} \mathrm{Bi}$ targets with ${ }^{50} \mathrm{Ti}$ and ${ }^{54} \mathrm{Cr}$ beams to produce elements $104,105,106$, and 107 . He measured spontaneous fission to identify these new elements. The results were heavily criticised by the Berkeley group. Firstly spontaneous fission is not a safe identification, secondly the Extra-Push 
model by Swiatecki forbids cold fusion of massive nuclear systems with large proton numbers. In 1980 we irradiated ${ }^{208} \mathrm{~Pb}$ with ${ }^{50} \mathrm{Ti}$ and created element 104 [6] observing the one-neutron evaporation channel (Fig. 2). Cold fusion was discovered. The way to heavy elements was open.
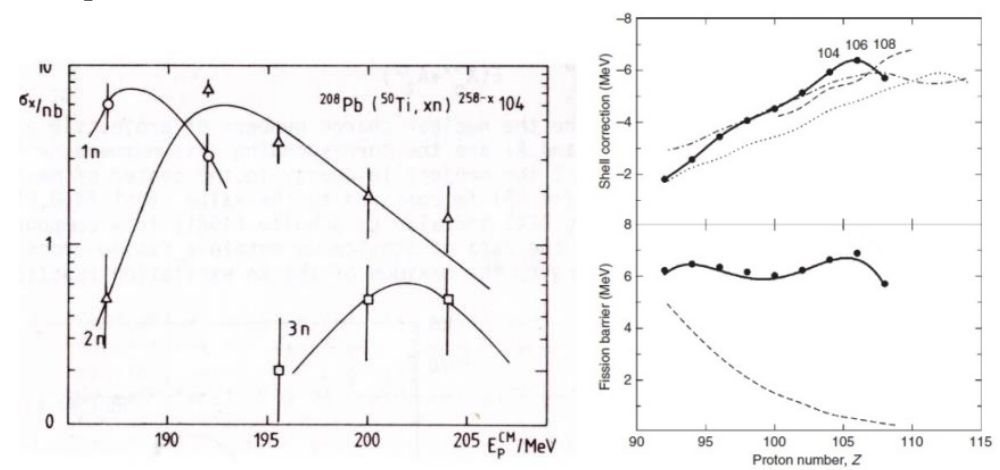

Fig. 2 left panel: excitation function for the production of element 104 in irradiations of ${ }^{208} \mathrm{~Pb}$ with ${ }^{50} \mathrm{Ti}$ [6], right panel upper part: shell correction energies for the doubly even $\mathrm{N}-\mathrm{Z}=48$ isotopes: dots and solid line experiment compared to calculations from Cwiok, dashed-dotted, Möller dotted, and dashed lines. Lower part: experimental fission barriers, the dashed line shows the liquid drop part of the barrier[7].

With the synthesis of element 106 and the discovery of element 108 at SHIP we proved the concept of super heavy nuclei. Due to the liquid drop model nuclei become unstable against fission at and beyond element 104 as shown on Fig 2. Here the liquid-drop fission barrier drops below $1 \mathrm{MeV}$. Spherical super-heavy elements were predicted for $Z=114$. Consequently a "sea of instability" beyond element 104 separated the "super heavy island" from the trans uranium elements. For element 108 fission half-lives of $1 \mu \mathrm{s}$ were predicted.

With element 108 as an $\alpha$-emitter with a half live of the order of milliseconds we discovered a new region of stability. The analysis of our experimental data shows an increase of shell stabilization for elements 106 and 108 to values of $-6 \mathrm{MeV}$. From these data we could construct experimental fission barriers as high as $6 \mathrm{MeV}$, due to shell stabilization. We had discovered a region of shell nuclei bridging the transuranium region and the spherical super heavy island [7]. Calculations of Möller and Sobiczewski show that this region is centred at $\mathrm{Z}=108$ and $\mathrm{N}=162$ and that the origin of the enhanced stability is a hexadecapole deformation. This is the confirmation of the idea of super heavy nuclei, pushed forward by Walter Greiner. In a Nobel Symposium in 1974 Aage Bohr commented a talk by Adam Sobiczewski: "What about the possibility of super heavy nuclei in other shapes which are stabilized by shell structure?"

This shell is the basis for the existence of the elements rutherfordium, $Z=104$, and beyond. These are now commonly called "super heavy elements".

\section{The future - SHE factories}

Fig 3. displays the production cross sections for the trans fermium elements in cold and hot fusion [8]. Cold fusion cross sections (in blue) drop fast towards the heaviest elements to a 
level of $22 \mathrm{fb}$ for nihonium, $\mathrm{Z}=113$. The reason is the fusion hindrance due to the fast increasing entrance fissility or, in other words, the fast increasing Coulomb repulsion between target and projectile. In hot fusion (in red) the entrance fissility does not change so fast. For heavy targets the relative change in the nuclear charge and consequently in the entrance is fissility small. The cross section is dominated by structure effects. The small bump is an indication for the $Z=114$ shell. The compound nuclei are close to the island of spherical SHE. Enhanced stability around $Z=114$ may show-up in the fusion cross sections but is not observed in the decay data. Cross sections are of the order of $10 \mathrm{pb}$.

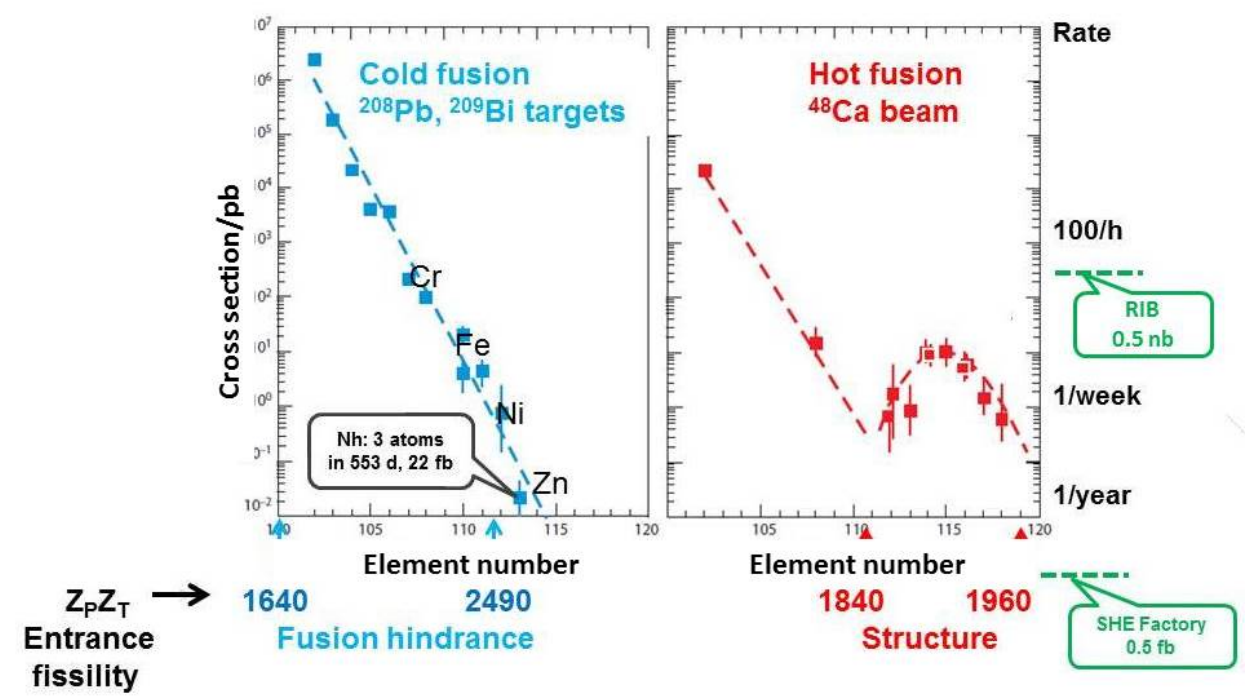

Fig. 3 Production cross sections for the trans fermium nuclei. Left panel: cold fusion, Right panel: hot fusion, the sensitivity limits for SHE factory and RIB are given per year of beam time. Hot fusion cross sections from [8].

At oganesson, element 118, the heaviest in sufficient amounts available target ${ }^{249} \mathrm{Cf}$ has been reached. Going beyond needs ${ }^{50} \mathrm{Ti}$ or ${ }^{54} \mathrm{Cr}$ projectiles. These are no magic nuclei: the prediction of fusion cross sections is a problem, they will be much smaller. First attempts to create element 120 have been made by Sigurd Hofmann (see this conference proceedings).

New prospects for SHE research will be opened up with the next generation SHE factories. Table 1 shows the beam intensities and annual doses available at present and with the future SHE-factories under construction. Already the present accelerators such as the UNILAC at GSI, the RIKEN RILAC, and the Dubna U400 cyclotron deliver beams of $6 \times 10^{12}$ ions/s on the average. With the beam time of $100 \mathrm{~d}$ to $300 \mathrm{~d}$ available for SHE research at JINR Dubna and RIKEN the sensitivity is 1 atom/10fb per year. SHE factories including JINR Dubna with the new DC-280 cyclotron, and RIKEN and SPIRAL2 at GANIL with new powerful linear accelerators and ion sources will have a factor of 10 to 100 more beam intensity. At GSI a new, compact super conducting LINAC dedicated to SHE research is under development. Taking into account the available beam time per year, the sensitivity is increased by a factor of about 50 , reaching a sensitivity of 5 atoms/fb and year. Other accelerator labs working on SHE research or planning such experiments: 
Argonne National Lab, Canberra, FRIB at Michigan State University, IMP Lanzhou, LBNL Berkeley, Tokai, and Jyväskylä will work on special topics including reaction studies, nuclear structure, chemistry, and atomic physics.

New in the field are Rare-Isotope, RI, facilities, SPIRAL2, FRIB, HIE ISOLDE, and FAIR-NUSTAR at GSI. The RI beam intensities close to stability are up to $10 \%$ s on the average. They drop to $10^{6} / \mathrm{s}$ five to ten isotopes away from stability. It turns out that the RI intensities for all schemes are of the same order of magnitude. FAIR intensities are higher for light beams such as neon and heavier. Because of the low beam intensity the use of RI beams for SHE research is rather limited at present. With an optimistic value of $10^{9}$ projectiles/s for isotopes close to stability the sensitivity is only 4atoms/nb per year. This is about six orders of magnitude less than achievable with the SHE factories with stable beams. Here it must be taken into account that SHE factories are dedicated to SHE research whereas RI facilities have a broad and competitive research program. First generation of experiments are reaction studies and isotope synthesis in the region up to rutherfordium (Fig. 3)

Table 1. Intensities and sensitivities for fusion reactions with stable and radioactive beams for SHE production [9] .

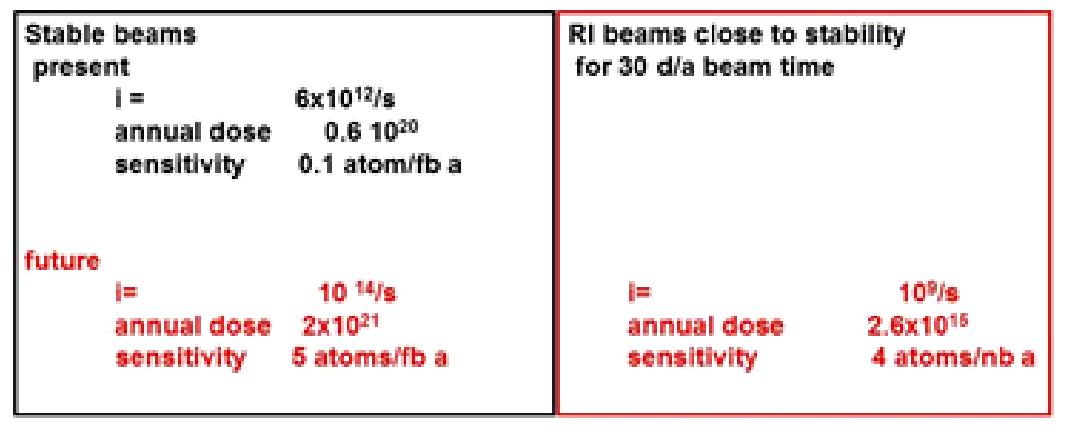

\subsection{Experiments and instrumentation}

The Discoveries of new elements beyond seaborgium have been made with the velocity filter SHIP at GSI, the gas filled separators DGFRS at JINR Dubna, and GARIS at RIKEN. While SHIP is a kinematic separator to separate atomic nuclei produced by heavy-ion fusion, gas filled separators separate heavy from light ions with bad resolution. They cannot separate fusion products from nuclei produced by incomplete fusion. The identification of SHEs is based on the measurement of correlated $\alpha$-decay chains of nuclei implanted into position sensitive surface barrier detectors. Decay chains ending in known nuclides allow the unambiguous assignment of individual, implanted nuclei.

Next generation SHE factories will open new possibilities for SHE research including atomic and more detailed nuclear studies as well as the discovery of new elements and isotopes. Certainly chemistry will play a major role in the future research programs and also rely on in-flight pre-separators to achieve highest sensitivity and clean conditions. A central goal is the exploration of the region of super heavies in the region of $Z=112$ to $Z=120$ and to approach the magic neutron shell $\mathrm{N}=184$. The $\alpha-\alpha$ correlation technique successfully 
applied to the discoveries of the heaviest elements will fail here as already became evident with the new trans-nihonium elements. Their ${ }^{\wedge}$-chains are not connected to the transuranium region. We should in addition be able to identify ß-decaying nuclei and those decaying by spontaneous fission. A next generation in-flight separator must include the capability of direct $\mathrm{A}$ and $\mathrm{Z}$ identification.

The next generation of in-flight separators includes gas filled separators and velocity filters with optimized transmission and separation quality to mention GARIS-II and the gas filled separator at Dubna under construction. SHELS at Dubna is a new velocity filter and within the Giessen-GSI-Manipal collaboration calculations for a compact and optimized velocity filter based on the experience with SHIP are under way (Fig. 4). The new generation of in-flight separators will be equipped with gas-filled stopping cells combined with high resolution experiments including Multiple-Reflection Time-of-Flight Mass Spectrometers (MR-TOF-MS) or Penning Trap systems. A first step in this direction is the SHIP-SHIPTRAP combination. At RIKEN an MR-TOF-MS system has been coupled to GARIS II. With this system the A,Z identification of astatine, polonium, and bismuth isotopes has been achieved by isobaric mass analysis at a mass resolving power of more than 100000 on the basis of about 10 atomic nuclei per isotope at minimum [10] First measurements of trans-uranium nuclei with A,Z identification have been performed by the same group. At GSI such a system has been operated successfully at FRS with a resolution of 500000 [11]. Isobaric A,Z identification and separation was achieved and the $\alpha$-decay of ${ }^{211} \mathrm{Po}$ at a detector behind the MR-TOF-MS was measured. With the MRTOF-MS method in contrast to the presently used identification by decay spectroscopy SHE are directly identified "still alive".

These systems can be operated in a separator mode and coupled to detector system such as Si surface barrier detectors, germanium arrays, or beta spectrometers. They can also be used to identify transfer products in large-scale survey experiments.

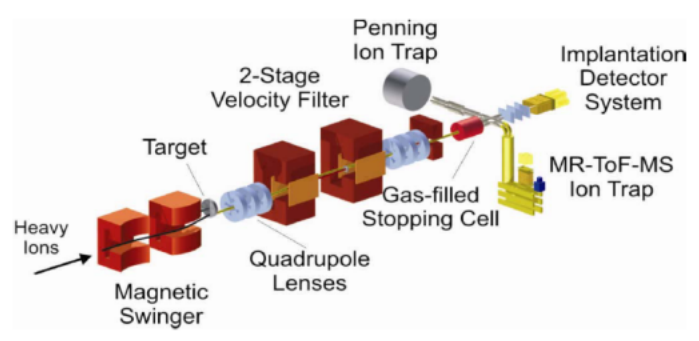

Fig. 4. A next generation compact velocity filter including a stopper cell and MR-TOF-MS or Ion Trap which can be used in combination with the commonly used implantation detector.

\section{Reaction studies}

Besides complete heavy ion fusion new reactions such as nuclear transfer will be investigated. Fig. 5 shows a prediction for transfer cross sections for the reaction ${ }^{238} U+$ ${ }^{248} \mathrm{Cm}$ by Zagrebaev and Greiner [12] compared to data from Schädel. They observed mendelevium as the heaviest element. Predicted cross sections drop fast, by about on order of magnitude per element. The power of nuclear transfer for the creation of new trans 
uranium isotopes is shown in Fig. 5 right. At SHIP in irradiations of ${ }^{248} \mathrm{Cm}$ with ${ }^{48} \mathrm{Ca}$ four new and trans uranium isotopes were found [13]. The identified isotopes are shaded. By far not all isotopes could be identified with our detection methods. With MR-TOF-MS a complete survey will be possible.
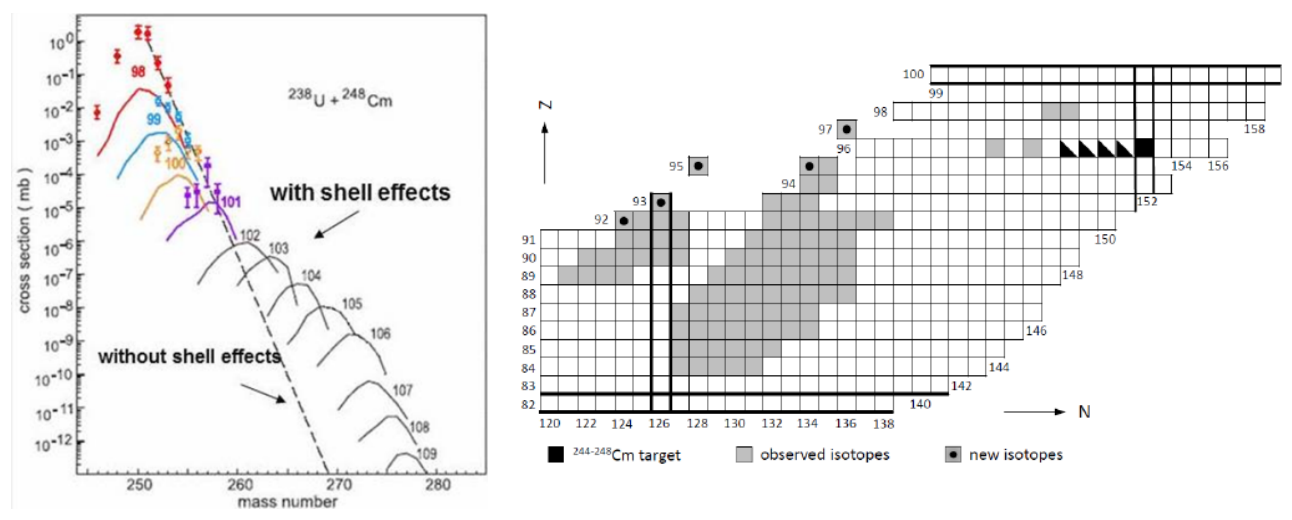

Fig. 5 Left panel experimental and predicted cross sections for heavy-element production by nuclear transfer [12], right panel new isotopes, marked by dots, created by in transfer reactions irradiating ${ }^{248} \mathrm{Cm}$ with ${ }^{48} \mathrm{Ca}[13]$.

Large-scale investigations of nuclear transfer reactions are under way e.g. with VAMOS at GANIL. The problem of in-beam methods is the sensitivity. As Fig. 5, left panel, shows, our main interest are the most exotic transfers created with small cross sections. Even very rare processes can be observed with MR-TOF-MS as it includes separation. The high resolution of MR-TOF-MS allows to separate isomers indicating the angular momenta of the final nuclei, crucial for the survival of SHE. In addition we aim to measure nuclear transfer under zero degree to create transfer products with low angular momenta to suppress prompt disintegration by fission.

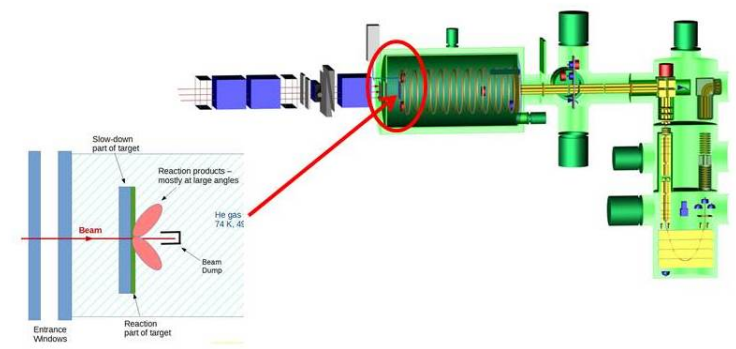

Fig. 6 Test setup for the investigation of transfer reactions at the FRS. The target is installed inside stopping cell (see inset left) can be irradiated with stable and radioactive beams at energies near Coulomb barrier [14].

A test setup for first transfer experiments is displayed in Fig. 6. It will be placed behind the FRS. Projectile fragments will be decelerated by energy degraders [14]. Stable and radioactive projectiles are available at near Coulomb-barrier energies. The target is 
placed inside the stopping cell where the reaction products are thermalized, extracted and guided to the MR-TOF-MS. A quadrupole mass filter will select the mass region of interest. For MR-TOF-MS operated in the separator mode the heavy nuclei can be directed to specialized detector system for detailed spectroscopy.

\section{Conclusion}

With the discoveries of nihonium and oganesson the era of fusion with magic nuclei ${ }^{208} \mathrm{~Pb}$ and ${ }^{48} \mathrm{Ca}$, theoretically substantiated by Walter Greiner has come to its end. With the discovery of the only by shell stabilization existing elements, rutherfordium and beyond, the prediction of super heavy nuclei existing by shell stabilization in the sea of liquid-drop instability has been proven.

To proceed, higher sensitivity is required, new reactions need being explored. Prospects for SHE research are opened by the new SHE factories with beam intensities increased by one to two orders of magnitude dedicated to SHE research. The search for trans-oganesson elements will be continued. More detailed structure investigations and SHE chemistry will play a major role in the future. The large facilities will be backed by laboratories with specialized research programs like in-beam spectroscopy and reaction studies. Studies of incomplete fusion, deep inelastic collisions including forward angles are under way and need being explored to small cross sections to see rare processes occurring with low probability. As a new development ion-catcher - ion-trap systems or MRTOF-MS will play a major role in SHE research. They will allow for direct identification of new isotopes and elements and in addition open new perspectives for atomic physics including laser spectroscopy of super heavy atomic nuclei.

I gratefully acknowledge support by the Giessen-GSI-Manipal collaboration $\mathrm{T}$. Dickel, H. Geissel, M. Gupta, S. Heinz, S. Hofmann, H.M. Devaraja, W.R, Plass, C. Scheidenberger, J. Winfield, M. Winkler.

\section{References}

[1] W. Greiner, U. Mosel, Z. f. Physik 222, 261 (1969)

[2] A. Sandulescu, W. Greiner, J. Phys. G 3, 189 (1977)

[3] R.K. Gupta, A. Sandulescu, W. Greiner, Z. Naturforsch. 32a. 704 (1077)

[4] IUPAC.org

[5] Nuclear. Phys. A 944, 1-690 ( 2015)

[6] G. Münzenberg et al., Actinides in perspective ed. N.E. Edelstein, Pergamon Press Oxford 1981, 223

[7] G. Münzenberg et al., Z. Phys. A 329, 49 (1987)

[8] Yu. Oganessian, roadmap 2011

[9] C. Scheidenberger priv. comm. 2012

[10] P. Schury et al. , Phys. Rev. C 95, 011305 (2017)

[11] T. Dickel et al. Nucl. Inst. and Meth. in Phys. Res. A 777, 172 (2015)

[12] V. Zagrebaev and W. Greiner, Phys. Rev. C 78, 034610 (2008)

[13] H.M. Devaraja et al. Phys. Lett. B 748, 199 (2015)

[14] T. Dickel et al, GPAC GSI 2017 